\title{
Pier Paolo Pandolfi wins Pezcoller-AACR International Award for Cancer Research
}

1. nuary, the Pezcoller Foundation and the American Association of Cancer Research (AACR) named Pier Paolo Pandolfi, professor at Harvard University Medical School and director of research at the Cancer Center and Genetics Program at Beth Israel Deaconess Medical Center, the winner of the 2011 International Award for Cancer Research. Pandolfi's successful research portfolio began with the discovery of the genetic basis of acute promyelocytic leukemia (APL), and his work in mouse models has led to highly successful treatments for the disease. The JCI recently spoke to Pandolfi about the award, his career, and his views on scientific research.

JCI: The Pezcoller-AACR award celebrates your career-long contribution to cancer research. What do you think is the most important discovery you've made?

Pandolfi: I think our APL story created a paradigm - how to move from a gene to a mouse model of cancer, to therapy - that could be, and has been, replicated for other cancers. I hope this award will be further stimulus to keep doing what we do; I really want to, within my lifetime, see this cancer beast defeated. And if I have further time, I'll try to fix something else.

JCI: Do you think your undergraduate training in philosophy shapes your approach to scientific questions?

Pandolfi: I think so; my approach is completely agnostic. I tend to think of myself as someone who doesn't know anything, and I work with my team so that we can discover together. One of the distinctive features of our approach is that we do creative science - we're trying to constantly challenge dogma. The ability to look at the logic and the big picture and to think outside the box - I inherited that kind of thinking from philosophy.

JCI: Science is a collaborative process, and your lab is a multidisciplinary one. How do you make that work?

Pandolfi: You have to enjoy the process of science - that's something you can feel in our lab. The lab is very active, very committed, but also very joyful. And the fact that we are in harmony in the lab was shown when we moved from MSKCC to Harvard three years ago: 30 people came with me. We came at once, rented two trucks, and it only took one week to be operational. We're all working toward the same goal, 30 of us thinking in a creative way, not only me. It's a great atmosphere when we meet because you have these creative ideas coming from left and right; it's really fun and inspiring.

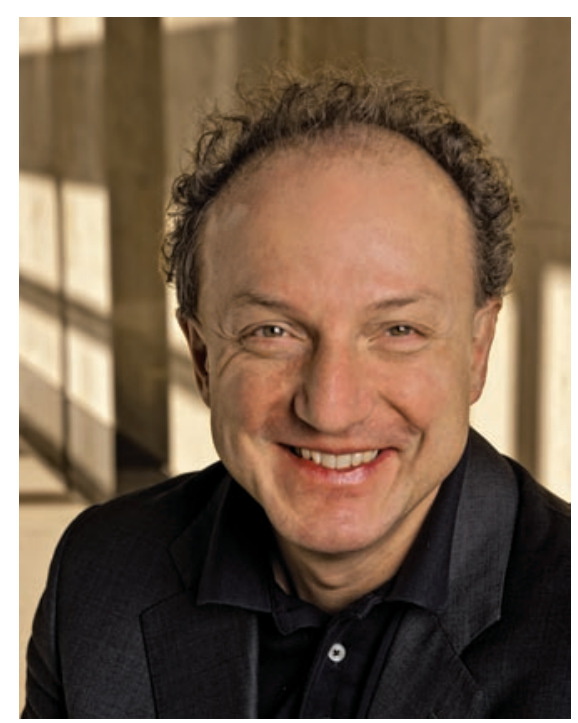

JCI: Your work pioneered the use of transgenic mice as models of cancer; how has that changed the field?

Pandolfi: Cancer is not one disease, but many. Our Cancer Center at Beth Israel Deaconess/Harvard is pushing - and so is the NCI - this idea of a "mouse hospital," that we should recreate many types of cancer in the mouse so we can rapidly find the best combination of drugs and translate that to the clinic. We think it's very important, because you can't address this issue by doing clinical trials in the old-fashioned phase I, phase II, phase III way . . . it will take you forever. If you take just one pathway - the PTEN, PI3K pathway - there are no less than 50 new molecules targeting it that could go in the clinic right away. If you consider breast or prostate cancer not as one disease, but as 15 or 25 , and you have 50 drugs, and you want to test them singly and in combination against each type of cancer ... it will take you forever. The question is not how to accelerate the speed of discovering drugs, but how to accelerate the testing of them. We think that the mouse models can be very informative to streamline these tests of efficacy.

JCI: Your lab recently described a new class of RNAs - competitive endogenous RNAs (ceRNAs). How do these change our understanding of the functional genome?

Pandolfi: We believe it's a very important discovery because it allows us to functionalize this space that we didn't understand. 50\% of the genome is transcribed, but only $2 \%$ makes protein, and there was no code, no language for understanding the rest. In terms of biomedical research, the impact is immense, because now you have all of these new entities; pseudogenes, long noncoding RNAs, microRNAs, that could be attributed a function. There are 20,000 pseudogenes, 10,000 long noncoding RNAs, and 20,000 genes in the human genome. Solely by functionalizing pseudogenes and long non-coding RNAs, you are almost tripling the size of the functional genome. We know that there are a number of translocations and rearrangements that are recurrent in cancer that don't impact any coding genes, and in the past we just swept this information under the carpet because we didn't know what to do with it. Now we have a way to attempt to predict the function. For us, it's been a stunning discovery; it never occurred to me that a messenger we were studying would have another function, and this forces us to reconsider the function of every gene we've ever studied because even a gene that codes for a protein might have another function that is driven not by that protein, but by the messenger.

JCI: You've been quoted as saying that you like to work on multiple different things simultaneously.

Pandolfi: I do that on purpose; creativity comes when you make a lot of strange, unexpected connections. I want to go to discovery with a free mind, and in this respect I love multidisciplinary approaches, the fusion of different cultures and methods. Socrates and his dialectic method are always in the back of my mind: opposing perspectives, asking and answering questions to stimulate critical thinking and to illuminate ideas. This is the core of contemporary cancer research and perhaps of any intellectual endeavor.

\section{Kathryn Claiborn}

